\title{
Peptidylarginine deiminase IV promotes the development of chemoresistance through inducing autophagy in hepatocellular carcinoma
}

\author{
Tingting Fan ${ }^{1}$, Changsong Zhang ${ }^{2,3}$, Ming Zong ${ }^{1}$, Qiudong Zhao ${ }^{3}$, Xue Yang ${ }^{3}$, Chong Hao ${ }^{3}$, Hui Zhang ${ }^{1}$,
} Shanshan Yu', Jinhu Guo ${ }^{1}$, Ruhan Gong ${ }^{1}$, Shasha Fan', Lixin Wei ${ }^{3^{*}}$ and Lieying Fan ${ }^{1 *}$

\begin{abstract}
Background: Peptidylarginine deiminase IV (PAD|4) is widely distributed in several tissues and the expression is correlated with many pathological processes. Chemotherapy remains a major treatment alternatively to surgery for a large number of patients at the advanced stage of hepatocellular carcinoma (HCC). However, the role of PADI4 in the chemoresistance of HCC has not been identified.

Methods: MTT and PI/Annexin V assay were employed to examine the proliferation and apoptosis of HCC cell lines. The expression of MDR1 is detected by Realtime PCR. GFP tagged LC3 expression vector and electron microscopy are utilized to demonstrate the occurrence of autophagy.

Results: We observed that the elevated PADI4 expression is associated with chemoresistance in HCC patients with TACE after surgery. In addition, we found that overexpression of PADI4 in HCC cell lines lead to the resistance to chemotherapeutic agents in vitro and in vivo. Interestingly, the HCC cells that overexpressed PADI4 were observed to undergo autophagy which was known as a protective mechanism for cells to resist the cell tosicity from chemotherapy. Autophagy inhibitor could effectively restore the sensitivity of HCC cells to chemotherapy in vitro and in vivo.
\end{abstract}

Conclusions: These results indicate that PADI4 may induce chemoresistance in HCC cells by leading autophagy.

Keywords: PADI4, Autophagy, Hepatocellular Carcinoma, Chemoresistance

\section{Background}

Peptidylarginine deiminases (PADs) enzymes distributed widely in mammals and their function is closely associated with the development and differentiation of the cells [1]. PADs can convert protein Arg residues to citrulline in a calcium and sulfhydryl group dependent manner, which play an important role in several physiological and pathological processes such as gene expression regulation, epithelial terminal differentiation and apoptosis [2].

\footnotetext{
* Correspondence: lixinwei@smmu.edu.cn; flieying@126.com

${ }^{3}$ Tumor Immunology and Gene Therapy Center, Eastern Hepatobiliary Surgery Hospital, Second Military Medical University, 225 Changhai Road, Shanghai 200438, China

'Department of Clinical Laboratory, Shanghai East Hospital, Tongji University Medical School, No. 150, Jimo Road, Shanghai 200120, China

Full list of author information is available at the end of the article
}

There are five types of PAD enzymes in human and mouse, including PADI1-4 and PAD6 [1,3]. PADI4 has been reported to express in several type of cells, including embryonic stem cells, leukocytes and breast cancer cells. Furthermore, PADI4 is also involved in the etiology of cancers and rheumatoid arthritis in human [4-6]. The results from immunohistochemistry demonstrates that PADI4 is expressed not only in various adenocarcinoma, but also in some non-adenocarcinoma tumors such as bone osteosarcoma, liver cholangiocellular carcinoma, liver hepatocellular carcinoma and lung squamous carcinoma [7]. These results indicates that PADI4 may play an important role in tumorigenesis [8].

Hepatocellular carcinoma (HCC) is a common malignant tumor in China, which surgical resection is considered as the most effective treatment. Chemotherapy remains to be a major treatment alternatively to surgery 
for a large number of patients at the advanced stage of HCC. However, chemoresistance in HCC is still a prominent obstacle for effective treatment of $\mathrm{HCC}$ with chemotherapy. Therefore, the underlying molecular mechanisms of HCC chemoresistance and how to overcome them is still a critical issue for us to identify. The role of PADI4 for chemoresistance of HCC cells has not been identified. In this study, we will investigate the role of PADI4 for chemoresistance in HCC and the potential mechanism will could be explored.

\section{Methods}

\section{Patients and tissue specimens}

Specimens of HCC tissues were obtained from 120 HCC patients who underwent hepatic resection at Eastern Hepatobiliary Surgery Hospital of Second Military Medical University from February 1998 to December 2010. These patients included 90 males and 30 females with a median age of 51 years (range: $31-73$ ). All tumors were histologically diagnosed as HCC according to the EdmondsonSteiner classification system. In all cases, H\&E-stained slides were re-examined independently by three experienced pathologists without any knowledge of clinical data. Written informed consent was obtained from each patient, and the protocol of the study was approved by the ethics committee of Second Military Medical University.

\section{Cell lines and reagents}

HCC cell lines including SMMC-7721 cells and Hep-G2 cells were cultured in Dulbecco's modified Eagle's medium (DMEM) (GIBCO, Invitrogen) with 10\% fetal bovine serum (FBS) at $37^{\circ} \mathrm{C}$ in a humidified atmosphere containing $5 \% \mathrm{CO}_{2}$. The chemotherapeutic agents: cisplatin and 5-fluorouracil (5-FU) were purchased from Qilu Pharmaceutical Co., Ltd. (JiNan, Shandong, China). 3-methyladenine (3-MA) (Sigma-Aldrich, Cat.M9281) was used at $10 \mathrm{mM}$.

\section{Adenoviral vectors}

The adenoviral vector expressing PADI4 under a cytomegalovirus promoter promoter-AdPADI4 was used to overexpress PADI4 in HCC cell lines. Ad-Vector (control adenovirus) infected group was used as controls in the subsequent experiments.

\section{HCC cell treatment}

To investigate the effect of PADI4 on the chemotherapy sensitivity of HCC cells, the cells were infected with AdPADI4 and then collected for the determinations of cell viability, apoptosis and the autophagy associated genes expression.

\section{Quantitative real-time PCR analysis for mRNA expression} RNA was isolated from tumor tissues, and blood samples. In brief, total RNA was extracted with TRIzol reagent, according to the protocol provided by the manufacturer. The quantity and quality of the RNA samples were measured carefully by spectrophotometer and electrophoresis. The first-strand cDNA was synthesised from $2 \mu \mathrm{g}$ of total RNA. Primer sequences of PADI4 for reverse transcriptionPCR (RT-PCR) reaction were forward (5' -CACAGCTCTG GTTGGCTTCA-3') and reverse (5' -CTGCACGTCCTTC AGCATCA-3') [9]. Primer sequences of MDR1 for reve rse transcription-PCR (RT-PCR) reaction were forward (5' CTGGTTTGATGTGCACGATGTTGG-3') and reverse (5' - TGCCAAG-ACCTCTTCAGCTACTG-3') [10]. Quantitative real-time PCR (qPCR) were carried out by using the Mx3000P QPCR System (Stratagene, USA). As an internal control for qPCR, $\beta$-actin mRNA expression was amplified from the same cDNA samples. All results were normalized to $\beta$-actin amplification. $C_{\mathrm{T}}$ values for triplicate reactions were averaged and relative expression was determined with the comparative $C_{\mathrm{T}}$ method, using average $C_{\mathrm{T}}$ values.

\section{MTT colorimetric assay}

In order to examine the effect of PADI4 on the chemosensitivity of HCC cells, HCC cells were seeded in 96well plates at a density of $1 \times 10^{4}$ cells/well and cultured in the medium containing chemotherapeutic agents with Ad-PADI4. The cell viability in each well was examined by a MTT (5 mg/ml, Sigma, Cat.M2003) colorimetric assay. The optical density (OD) value at $490 \mathrm{~nm}$ of each sample was measured using a plate reader. The data was expressed as mean $\pm \mathrm{SD}$.

\section{Cell apoptosis assay}

HCC cells $\left(2 \times 10^{5}\right.$ cells/well $)$ were cultured in 6-well plates to $70-80 \%$ confluence. The cells were then treated with chemotherapeutic agents for $8 \mathrm{~h}$ in the absence and presence of MSC conditioned medium. In a subset of experiments, 3-MA (5 mM) was used to block autophagy. $\mathrm{PI} /$ Annexin V-PE assay was used to measure apoptotic cells by flow cytometry according to the manufacturer's instruction (Keygen Biotech. Co., Ltd, Nanjing, Jiangsu, China, Cat.KGA108). Briefly, cells collected by trypsinization were washed trice with ice cold phosphate-buffered saline (PBS). Cells were then incubated in $300 \mu \mathrm{L}$ of $1 \times$ binding buffer containing $5 \mu \mathrm{L}$ Annexin V and $5 \mu \mathrm{L}$ PI for $30 \mathrm{~min}$ at room temperature in the dark. Apoptosis of cells was measured on a BD FACSAria flow cytometer (Becton Dickinson, Lincoln Park, NJ). At least 30,000 gated events were acquired from each sample. Results are expressed as the percentage of apoptotic cells (PI and Annexin $\mathrm{V}$ positive) in the gated cell population.

\section{Transient transfection and identification of autophagy}

GFP tagged LC3 expression vector has recently been utilized to demonstrate the occurrence of autophagy. SMMC- 
7721 and Hep-G2 cells were seeded $\left(1 \times 10^{4}\right.$ cells/well $)$ in 96-well plates overnight. GFP-LC3 expressing plasmids were transiently transfected into cells using the Fugene HD transfection reagent (Roche, Cat.04709705001) according to the manufacturer's instruction. After being cultured for $24 \mathrm{~h}$ to ensure expression of GFP-LC3, the cells were treated with Ad-PADI4 for $8 \mathrm{~h}$. At the end of each experiment, autophagy was detected by counting the percentage of cells with GFP-LC3-positive dots under fluorescence microscope (Olympus IX71). A minimum of 200 cells were counted in each sample. The experiment was conducted in triplicate.

\section{Electron microscopy}

HCC cells were sequentially fixed with $2.5 \%$ glutaraldehyde acid in 0.1 M PBS buffer ( $\mathrm{pH} 7.4$ ) for $2 \mathrm{~h}$, incubated in $1 \%$ osmium tetroxide in $0.1 \mathrm{M}$ PBS buffer ( $\mathrm{pH}$ 7.4) for $2-3 \mathrm{~h}$, dehydrated in solutions of ethanol and acetone, embedded in Araldite, and finally solidified. Sections $(50-60 \mathrm{~nm})$ were cut on a LKB-I ultramicrotome and picked up on copper grids. After being post-stained with uranyl acetate and lead citrate, sample sections were observed with a Philips CM-120 TEM (Philips).

\section{Western blot analysis}

HCC cell lines were washed in PBS and lysed in RIPA buffer with $1 \mathrm{mM}$ PMSF on ice. Cell lysates were centrifuged $(12,000 \mathrm{rpm}, 10 \mathrm{~min})$ at $4^{\circ} \mathrm{C}$, the protein supernatant was transferred into new tubes. The concentration of the protein samples was determined with BCA Protein Assay Kit (Pierce, USA). A $20 \mu \mathrm{g}$ sample of the total protein was resolved using 12\% SDS-PAGE and transferred onto PVDF membranes. The membranes were blocked in Trisbuffered saline containing Tween 20 (TBST) with 5\% nonfat milk at room temperature for $2 \mathrm{~h}$. Primary antibodies to detect PADI4(1:1000, Abcam, USA) and LC-3(1:1000, Novus Biologicals, Inc) were incubated overnight with the membranes at $4^{\circ} \mathrm{C}$. Membranes were incubated with horseradish peroxidase (HRP)-conjugated anti-rabbit secondary antibodies (1:8000, Dako, USA), and proteins were detected by enhanced chemiluminescence (ECL) (Beyotime, USA). GAPDH was used as the internal control to normalize the loading materials.

\section{Animal model}

All procedures involving animals were performed in accordance with the institutional animal welfare guidelines of Second Military Medical University. Subcutaneous implantation of HCC cells was performed in armpit areas of nude mice. Mice were examined three times per week. Tumor growth was evaluated by measuring the length and width of the tumor mass. Animals were sacrificed and tumors were removed at the end of the experiment. Tumor masses were weighed and analyzed by histology.

\section{Short Hairpin RNA (shRNA) synthesis and transient transfection}

The shRNA sequences of PADI4, Atg7 and Beclin7 were designed using Oligoengine software and verified by nucleotide BLAST searches. The candidate sequences and a scrambled sequence with no significant homology were listed in Additional file 1: Table S1. The recombinant virus was packaged using Lentivector Expression Systems (Shanghai GeneChem, Shanghai). Cells $\left(1-3 \times 10^{6}\right)$ growing to $50 \%-60 \%$ confluence in $10 \mathrm{~cm}$ petri dishes were transfected with shRNA sequences. Cells were observed under a fluorescence microscope and harvested $48 \mathrm{~h}$ after transfection.

\section{Statistical analysis}

All data were generated without knowledge of the clinical status of the samples analyzed by SPSS 18.0 software (SPSS, Inc., Chicago, USA). Comparison was done with $t$ test (unpaired or paired). All $\mathrm{P}$ values presented were two-sided, and a P value of less than 0.05 was considered statistically significant.

\section{Results}

The elevated PADI4 expression is associated with chemoresistance in HCC with Transcatheter arterial chemoembolization (TACE)

We collected $5 \mathrm{ml}$ vein blood samples before TACE and after TACE in HCC patients with partial hepatectomy. Chemoresistance were detected by MDR1 mRNA expression in blood samples by using Realtime-PCR. We found that elevated expression of MDR1 after TACE was detected in $72.5 \%$ of HCC patients $(87 / 120)$, and lower expression of MDR1 was detected in $27.5 \%$ of HCC patients (33/120) after TACE. Here, MDR1 (+) group means elevated expression of MDR1, and MDR1 (-) group means lower expression of MDR1 after TACE treatment for HCC patients with partial hepatectomy. According to the MDR1 results, all $120 \mathrm{HCC}$ patients were divided into two groups: the chemoresistance group-MDR1 (+) group, and chemosensitivity group-MDR1 (-) group (Figure 1A). However, there are no significant difference in clinicopathologic variables between MDR1 (+) group and MDR1 (-) group (Additional file 1: Table S2).

Time zero of recurrence-free survival of patients treated with TACE is the date of TACE. All patients underwent ultrasound, computed tomography scan or magnetic resonance imaging of the abdomen once every 2 months after treatment. Kaplan-Meier analysis indicated that the recurrence-free survival of MDR1 (+) group was shorter than MDR1 (-) group (median: 6 months vs 9 months, respectively; $P<0 \cdot 0001$; HR: 

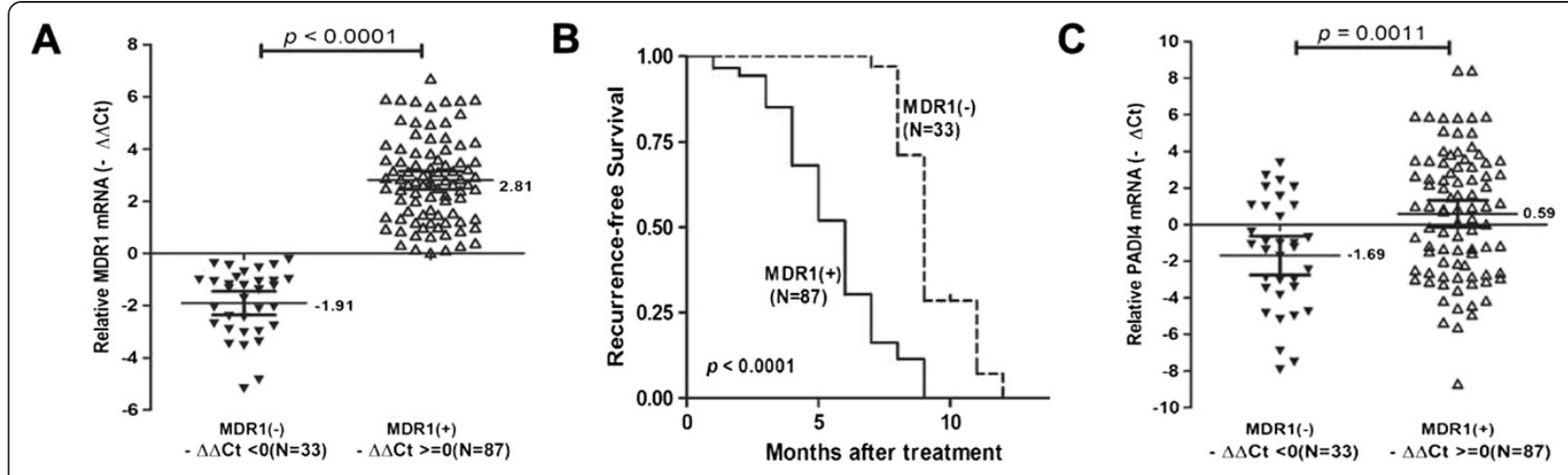

Figure 1 The elevated PADI4 mRNA expression in MDR1(+) HCC patients with TACE. (A) The MDR1 mRNA expression were detected in blood samples before and after TACE. - $\Delta \Delta C \mathrm{Ct},-\left(\Delta \mathrm{Ct}_{\text {before TACE }}-\Delta \mathrm{Ct}_{\text {after TACE); }} \mathrm{MDR}(+)\right.$ :- $\Delta \Delta \mathrm{Ct}>=0$; $\mathrm{MDR}(-)$ :- $\Delta \Delta \mathrm{Ct}<0$. (B) The progression-free survival (PFS) after transcatheter arterial chemoembolization (TACE) in partial hepatectomy for hepatocellular carcinoma determined by Kaplan-Meier analysis. (C) The PADI4 mRNA expression in HCC tumor tissues associated with chemoresistance. - $\Delta \Delta C \mathrm{Ct},-\left(\Delta \mathrm{Ct}_{\text {before }}\right.$ TACE $-\Delta C \mathrm{Ct}_{\text {after }}$ TACE); $\mathrm{MDR}(+)$ :$\Delta \Delta \mathrm{Ct}>=0 ; \operatorname{MDR}(-):-\Delta \Delta \mathrm{Ct}<0$.

6.339, 95CI\%: 3.742-10.74, Figure 1B). Interestingly, we found that PADI4 mRNA expression of tumor tissues was higher in MDR1 $(+)$ group (Mean ${ }_{-\Delta \mathrm{Ct}} \pm \mathrm{SD}=0.59 \pm 0.37$ ) than that in MDR1 (-) group (Mean $-\Delta \mathrm{Ct} \pm \mathrm{SD}=-1.69 \pm$ 0.53). PADI4 expression of HCC tissues was significantly difference between chemoresistance patients and chemosensitivity patients $(p=0.0011$; Figure $1 C)$.

\section{PADI4 enhanced chemoresistance of hepatocellular carcinoma cells}

In order to investigate the influence of PADI4 on the chemoresistance in HCC cells, we observed the effect of chemotherapeutic agent 5-Fu on morphological changes of HCC cell line-SMMC7721 cells. The expression of PADI4 in HCC cell lines was examined by western blot and Ad-PADI4 was used to overexpress PADI4 in HCC cell lines (Additional file 2: Figure S1). As shown in Figure 2A, 5-Fu could effectively inhibit the growth of SMMC7721 cells. Once PADI4 was overexpressed in SMMC7721 cells, the inhibition effect induced by 5 -Fu was attenuated. We also observed the role of PADI4 on the proliferation of SMMC7721 and Hep-G2 cells when they were exposed to chemotherapeutic agent. The results demonstrated that overexpression of PADI4 significantly improved the chemoresistance capability in hepatocarcinoma cell lines compared with control groups (Figure 2B). In addition, we detected the effect of PADI4 on the apoptosis of HCCs when they were exposed to chemotherapeutic agent. The results showed that PADI4 effectively decreased the apoptosis of SMMC7721 cells induced by 5-Fu (Figure 2C). We further examined the PADI4-induced chemoresistance in HCC cells in the nude mouse model. As shown in Figure $3 \mathrm{~A}$ and $\mathrm{B}$, compared with control groups, PADI4 induced the resistance to chemotherapy in HCC cells in vivo.
Taken together, these data suggested that overexpression of PADI4 could effectively induce the chemoresistance in HCC cells.

PADI4 induced autophagy in hepatocellular carcinoma cells Autophagy has been reported to contribute to chemoresistance in HCC cells [11]. Therefore, we speculated that PADI4 might lead to an incidence autophagy in HCC cells. A vector that expresses encoding GFP-LC3 was transmitted into SMMC7721 cells to detect the occurrence of autophagy. We determined the incidence of autophagy in HCC cells when the punctuate GFP fluorescence was observed in a diffused GFP fluorescence. The results showed that SMMC7721 with overexposed PADI4 exhibited a high percentage of punctuate GFP, which indicated the occurrence of autophagy, while the fluorescence in control groups remained to be diffused (Figure 4A and B). Transmission electron microscopy was employed to confirm the autophagy in SMMC7721 cells. As shown in Figure 4C, SMMC7721 cells with overexpressed PADI4 demonstrated a marked autophagosomes accumulation, which indicated the incidence of autophagy. Furthermore, we examined the expression of LC3-I and LC3-II in HCC cells by western blot. The level of LC3-I and LC3-II increased in PADI4 overexpression groups and in hypoxia treated group, which indicated that PADI4 could induce autophagy in HCC cells (Figure 4D).

\section{Inhibition of autophagy restored the sensitivity of HCC cells to chemotherapy}

In order to confirm the role of autophagy in enhancing the chemoresistance of HCC cells, we observed the recovery of HCC cell sensitivity to chemotherapy leading by autophagy inhibitors. The 3-MA is used as autophagy inhibitor, which blocks the formation of autophagosomes [12]. As shown in Figure 5, the results demonstrated 
A

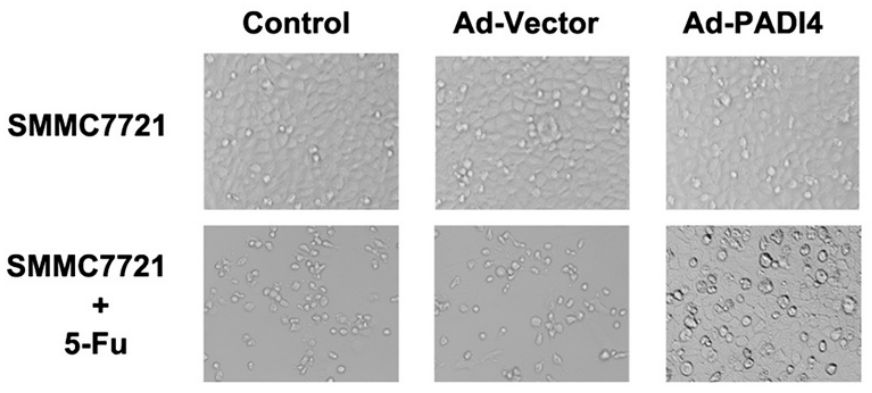

B
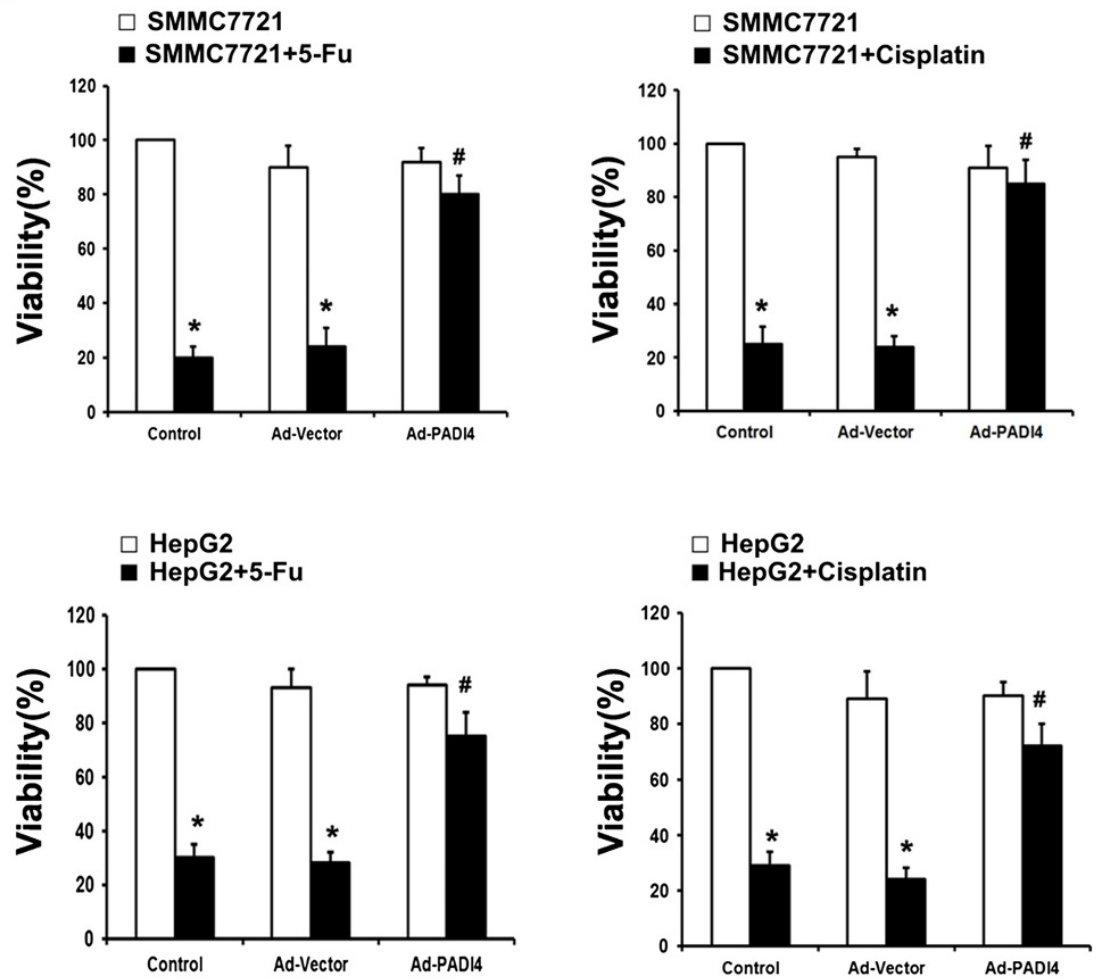

C
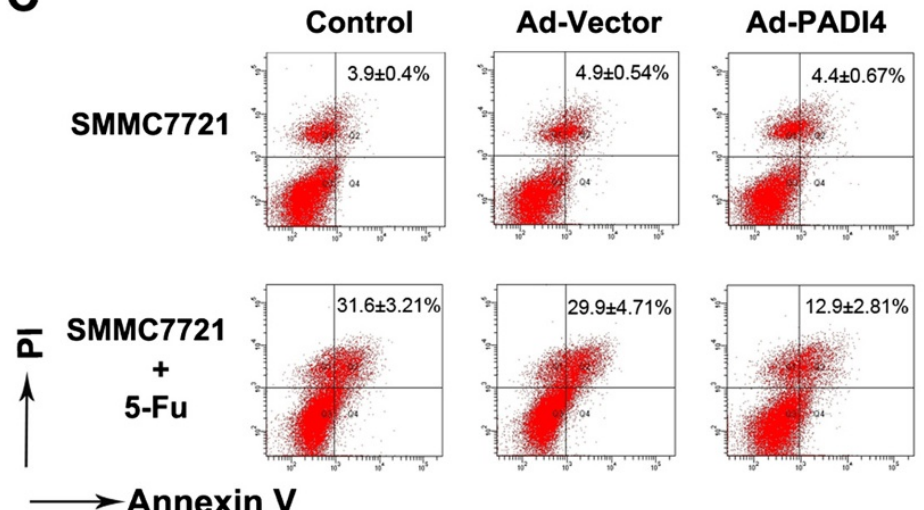

Figure $\mathbf{2}$ (See legend on next page.) 
(See figure on previous page.)

Figure 2 PADI4 enhanced chemoresistance of hepatocellular carcinoma cells in vitro. (A) SMMC-7721 cells were treated with 5-Fu (120 $\mu \mathrm{g} / \mathrm{mL}$ ) with overexpression of PADI4 or not. The morphology of the cells was observed by microscope. (B) PADI4 was overexpressed in SMMC-7721 or HepG2 cells and the cells $\left(1 \times 10^{4} /\right.$ well) were cultured in a $96-$ well plate with an existence of 5 -Fu $(120 \mu \mathrm{g} / \mathrm{mL})$ or cisplatin $(8 \mu \mathrm{g} / \mathrm{mL})$ for 24 hours. MTT was employed to examine the viability of SMMC-7721 or Hep-G2 cells. (C) PADI4 was overexpressed in SMMC-7721 cells and the cells were cultured in a 6-well plate with an existence of 5-Fu $(120 \mu \mathrm{g} / \mathrm{mL})$ for 24 hours. Flow cytometry was used to measure apoptosis of the cells. ( ${ }^{*} \mathrm{Compared}$ with the group that untreated with chemotherapy drugs, $\mathrm{P}<0.05$; \#Compared with the group that treated with chemotherapy drugs, $\mathrm{P}<0.05$ )

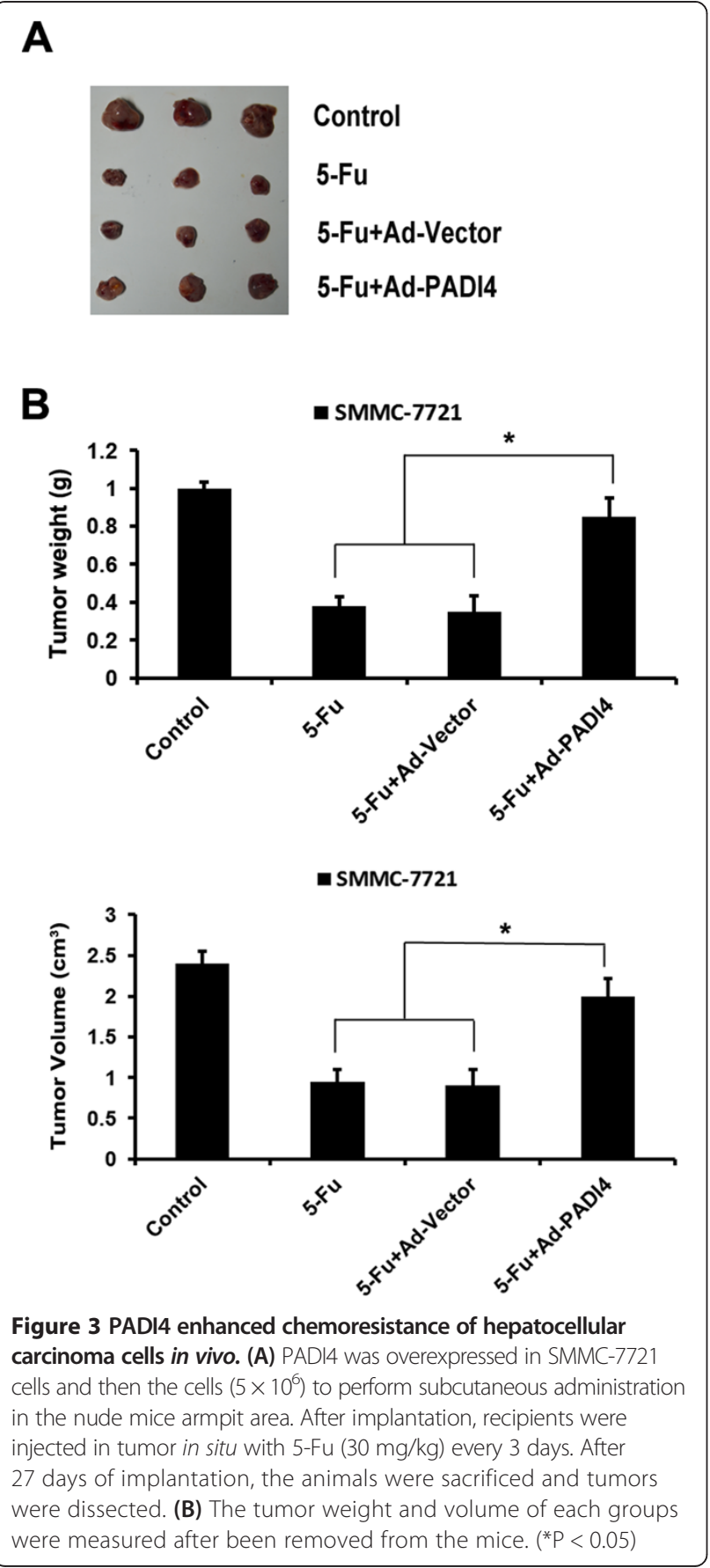

that 3-MA could significantly restore the chemotherapeutic sensitivity in HCC cells. Beside that, another autophagy inhibitor-CQ demonstrated a same effect as 3-MA (Additional file 3: Figure S2). Furthermore, we also employed shRNA-ATG7 or shRNA-Beclin1 to inhibit autophagy in HCC cells. As shown in Additional file 4: Figure S3, shRNA-ATG7 and shRNA-Beclin1 could effectively inhibit the upregulation of ATG7 and Beclin1 in HCC cells during cultured in hypoxia condition. The inhibition of ATG7 or Beclin1 respectively in HCC cells effectively diminished the protection of autophagy on chemoresistance.

\section{Discussion}

Hepatocellular carcinoma (HCC) is a common malignant tumor in China, which surgical resection is considered as the most effective treatment. Chemotherapy remains to be a major treatment alternatively to surgery for a large number of patients at the advanced stage of HCC. However, chemoresistance in HCC cells is still a prominent obstacle for effective treatment of $\mathrm{HCC}$ with chemotherapy.

Multiple drug resistance (MDR) phenotype, which almost constantly expressed in HCC, is generally considered as a key cause of poor outcome in HCC chemotherapy $[13,14]$. MDR is associated with overexpression of the MDR1 gene and the expression of a transmembrane glycoprotein of 150-180 kDa membrane phosphoglycoprotein (P-gp). As a drug pump, it confers cancer cell resistance to a broad range of structurally and functionally diverse chemotherapeutic drugs $[15,16]$. It is well documented that MDR has frequently been associated with elevated expression level of the MDR1/P-gp in hepatocellular carcinoma cell lines $[17,18]$.

Protein citrullination had recently become an interest area in some types of cancer. PADI4 was a transcriptional coregulator that catalyzes the calcium-dependent conversion of specific arginine residues in proteins to citrulline, and had also been proposed to "reverse" epigenetic histone modifications [19]. PADI4 expression was also significantly detected in some non-adenocarcinoma tumors such as liver cholangiocellular carcinoma and hepatocellular carcinoma, but the levels of PADI4 expression by immunohistochemistry or western blot analysis were very different in various malignant tumor tissues and blood 


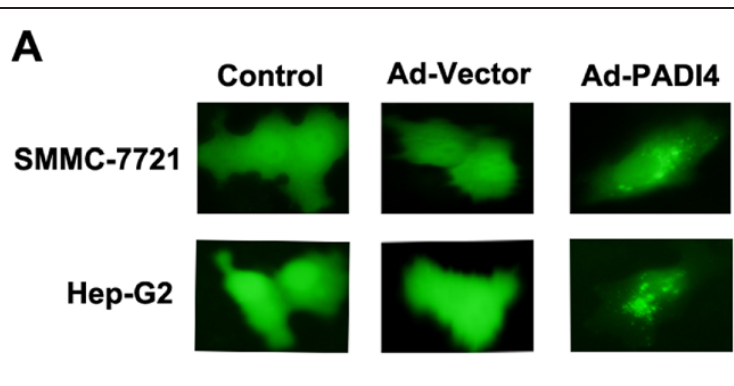

B
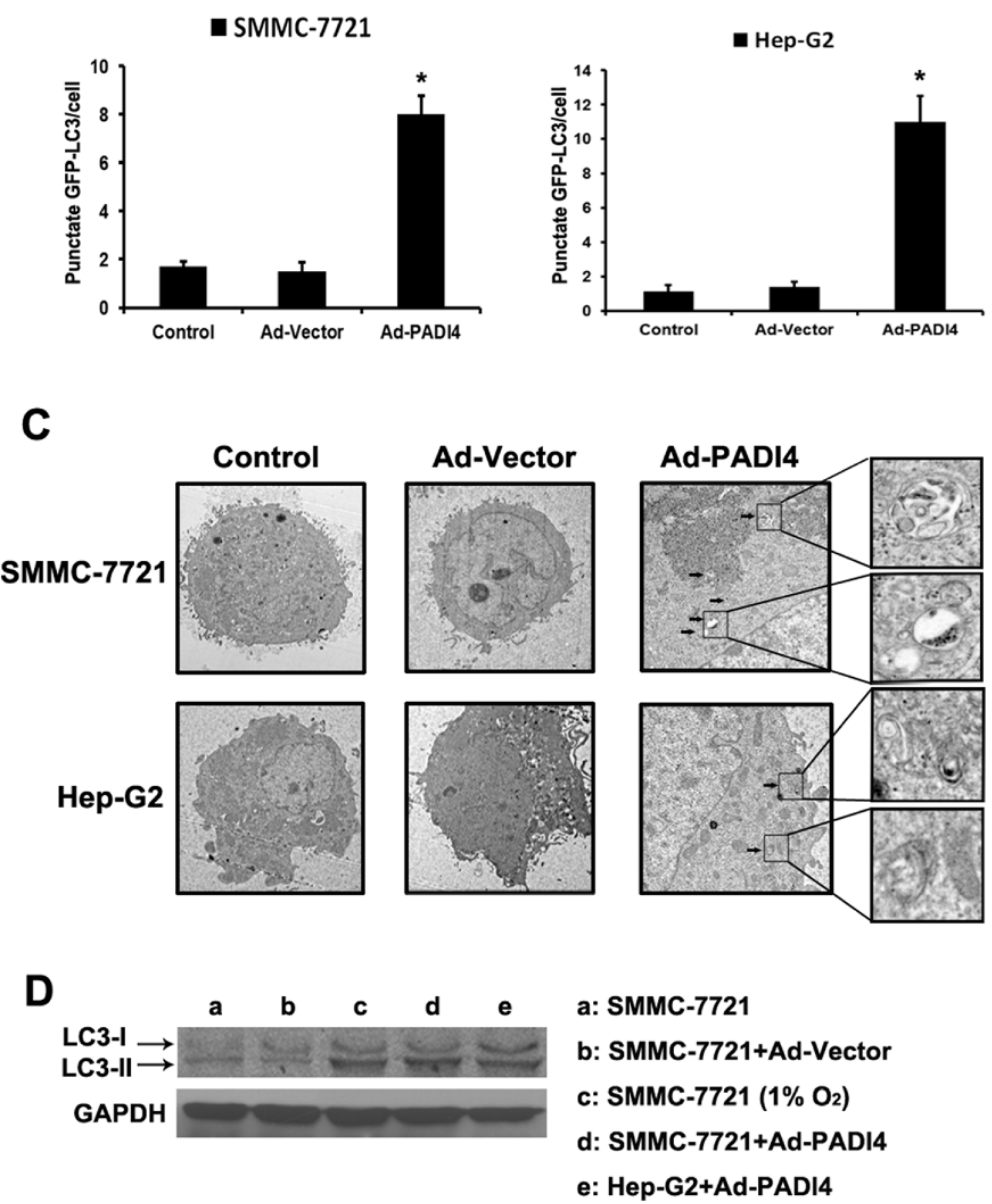

Figure 4 PADI4 induced autophagy in hepatocellular carcinoma cells. (A) GFP-tagged LC3 plasmid was transfected into SMMC-7721 cells, after 24 hours transfection, the cells were incubated with Ad-PADI4. Fluorescence microscope was used to observe the punctate GFP-LC3 in the cytoplasm. (B) The number of punctate GFP-LC3 in each cell of SMMC-7721 and HepG2 was counted and at least 100 cells were included for each group. (C) Electron micrographs was exployed to examine the autophagic vacuoles ultrastructure in the cytoplasm of SMMC-7721 and HepG2 cells which PADI4 were overexpressed. Magnification, $\times 10,000$. (D) Western blot was used to analysis the expression of LC3-I and LC3-II in SMMC7721 and HepG2 cells. a-d: SMMC-7721 in different treatment conditions; e: HepG2 treated with Ad-PADI4. GAPDH expression was used as control.

[20,21]. In this study, we found that the elevated PADI4 expression is associated with chemoresistance in HCC patients with TACE after surgery. Therefore, we explore the role of PADI4 in chemoresistance of HCC cells. We found that overexpression of PADI4 in HCC cell lines could lead to the resistance to chemotherapeutic agents in vitro and in vivo.
Autophagy is an evolutionarily conserved catabolic process, which serve as a survival mechanism in a nutrient deficient environment not only for prokaryotic but also for eukaryotic cells [21,22]. The autophagy associated pathway consists three steps: formation of autophagosome, lysosomal fusion with the autophagosome, and lysosomal degradation to produce precursor molecules, 

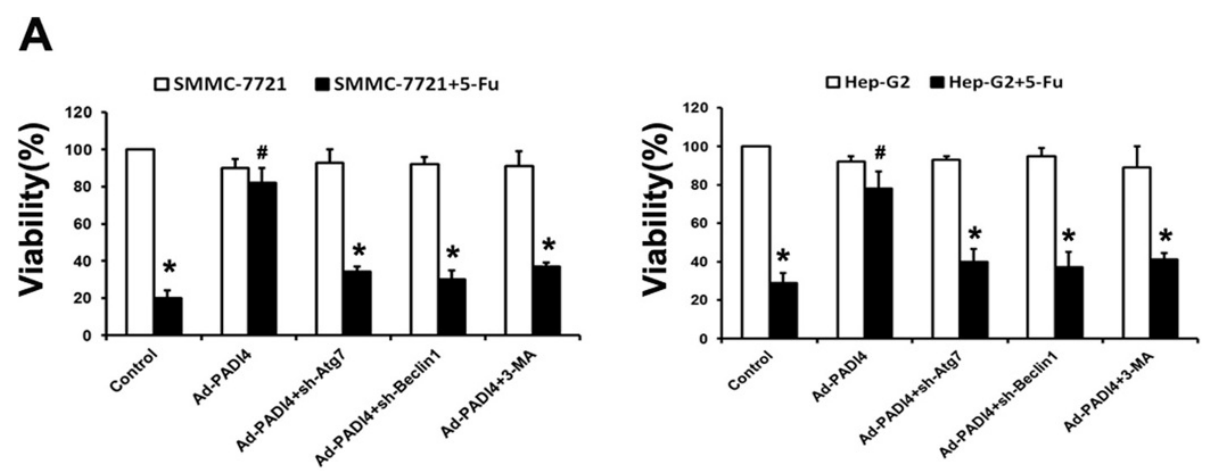

B
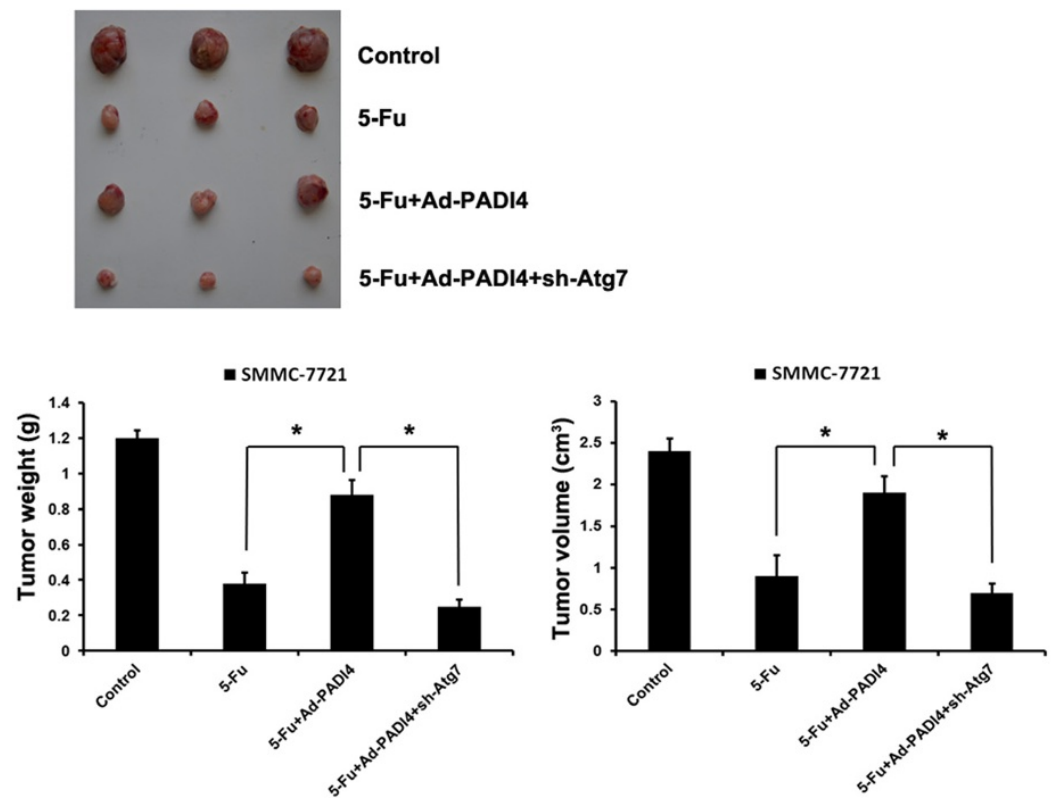

Figure 5 Inhibition of autophagy restored the sensitivity of HCC cells to chemotherapy. (A) SMMC-7721 and Hep-G2 cells $\left(1 \times 10^{4} /\right.$ well) that overexpressed PADI4 were cultured in a 96-well plate with an existence of 5-Fu (120 $\mu \mathrm{g} / \mathrm{mL})$ for 24 hours. The occurrence of autophagy was inhibited by autophagy inhibitor-3-MA or sh-Atg7 and sh-Beclin1. MTT was used to detect the viability of the cells. (B) PADI4 was overexpressed in SMMC-7721 cells. The occurrence of autophagy was inhibited by sh-Atg7. Then the cells $\left(5 \times 10^{6}\right)$ were performed subcutaneous administration in the nude mice armpit area. After implantation, recipients were injected in tumor in situ with $5-\mathrm{Fu}(30 \mathrm{mg} / \mathrm{kg})$ every 3 days. After 27 days of implantation, the animals were sacrificed and tumors were dissected. The tumor weight and volume of each groups were measured after been removed from the mice. ${ }^{*}$ Compared with the group that untreated with chemotherapy drugs, $\mathrm{P}<0.05$; \#Compared with the group that treated with chemotherapy drugs, $\mathrm{P}<0.05)$

such as amino acids fatty acids and other precursor molecules, to be reutilized for maintaining cellular homeostasis and facilitating cell survival [23-26]. Autophagy has been reported to be associated with several physiological and pathological processes, including cell differentiation, tumorigenesis and adaptation to changed environmental conditions [27]. However, the role of authphagy in tumorigenesis is still controversial. On one hand, some studies reported that autophagy is essential for the survival of cancer cells not only in cancer cells but also in cancer stem cells $[11,28]$. On the other hand, prolonged autophagy will lead to non-apoptotic type II programmed cell death $[29,30]$.

In previous studies, we have demonstrated that autophagy decreases the sensitivity of hepatoma cells to chemotherapeutic agents by affecting their apoptotic potential [11]. In addition, we have shown that autophagy activated by hypoxia mediates the tolerance of hepatocellular carcinoma cells to nutrient deprivation, which is dependent on the activity of Beclin 1 [19]. Wang, et al. found that PADI4 regulates the mTORC1 signaling pathway and PADI inhibitors are potential anticancer 
reagents that activate tumor suppressor gene expression alone or in combination with HDAC inhibitors [31].

Interestingly, our study indicated that HCC cells with overexpressed PADI4 were observed to undergo autophagy, which is known as a protective mechanism for cells to resist the cell toxicity from chemotherapy. Autophagy inhibitor could effectively restore the sensitivity of HCC cells to chemotherapy in vitro and in vivo. These results indicate that PADI4 could induce chemoresistance in HCC cells by leading autophagy.

\section{Conclusions}

Taken together, PADI4 could play an important role in inducing chemoresistance in HCC cells, and the expression of PADI4 of tumor tissues in HCC patients could be used as a prognostic indicator. However, this remains a speculation and more studies are needed in the future to elucidate the exact molecular mechanisms of PADI4 in $\mathrm{HCC}$.

\section{Additional files}

Additional file 1: Table S1. Sequence of the oligonucleotides for shRNA construct-making assays. Table S2. Correlations Between MDR1 Expression and Clinicopathologic Variables of HCC.

Additional file 2: Figure S1. The original expression and overexpression of PADI4 in HCC cell lines: Western blot was used to analysis the expression of PADI4 in SMMC7721 and HepG2 cell lines. GAPDH expression was used as control.

Additional file 3: Figure S2. Inhibition of autophagy restored the sensitivity of HCC cells to chemotherapy: SMMC-7721 and Hep-G2 cells $\left(1 \times 10^{4} /\right.$ well $)$ that overexpressed PADI4 were cultured in a 96-well plate with an existence of $5-\mathrm{Fu}(120 \mu \mathrm{g} / \mathrm{mL})$ for 24 hours. The occurrence of autophagy was inhibited by autophagy inhibitor-CQ. MTT was used to detect the viability of the cells.

Additional file 4: Figure S3. The knowndown efficacy of shRNA-ATG7 and shRNA-Beclin1: Realtime PCR was employed to examine the knowndown efficacy of shRNA-ATG7 and shRNA-Beclin1 in HCC cells when cultured in hypoxia condition.

\section{Abbreviations}

PADs: Peptidylarginine deiminases; TACE: Transcatheter arterial

chemoembolization; HCC: Hepatocellular carcinoma.

\section{Competing interests}

The authors declare that they have no competing interests.

\section{Authors' contributions}

TTF: Conception and design, data analysis and interpretation, manuscript writing; CSZ: Conception and design, collection and/or assembly of data, manuscript writing; MZ: Conception and design, data analysis and interpretation; QDZ: Provision of study material or patients, collection and/or assembly of data, data analysis and interpretation; $\mathrm{XY}$ : Provision of study material or patients, collection and/or assembly of data; $\mathrm{CH}$ : Provision of study material or patients, collection and/or assembly of data; HZ: Provision of study material or patients, collection and/or assembly of data; SSY: Administrative support, collection and/or assembly of data; JHG: Collection and/or assembly of data, data analysis and interpretation; RHG: Data analysis and interpretation; SSF: Data analysis and interpretation; LXW: Conception and design, financial support, data analysis and interpretation, final approval of manuscript; LYF: Conception and design, financial support, data analysis and interpretation, final approval of manuscript. All authors read and approved the final manuscript.

\section{Acknowledgements}

This study was supported by the Natural Science Foundation of China (Grant No: 81372212, 81373203), Shanghai Municipal Science and Technology Commission (No. 12XD1404300, No.11DZ1973802), the Natural Science Foundation of Jiangsu Province (No. BK2011251, BL2013012).

\section{Author details}

${ }^{1}$ Department of Clinical Laboratory, Shanghai East Hospital, Tongji University Medical School, No. 150, Jimo Road, Shanghai 200120, China. ${ }^{2}$ Clinical Oncology Laboratory, Changzhou cancer Hospital of Soochow University, Changzhou 213001, China. ${ }^{3}$ Tumor Immunology and Gene Therapy Center, Eastern Hepatobiliary Surgery Hospital, Second Military Medical University, 225 Changhai Road, Shanghai 200438, China.

Received: 9 April 2014 Accepted: 7 August 2014

Published: 26 August 2014

\section{References}

1. Vossenaar ER, Zendman AJ, van Venrooij WJ, Pruijn GJ: PAD, a growing family of citrullinating enzymes: genes, features and involvement in disease. Bioessays 2003, 25(11):1106-1118.

2. Gyorgy B, Toth E, Tarcsa E, Falus A, Buzas El: Citrullination: a posttranslational modification in health and disease. Int J Biochem Cell Biol 2006, 38(10):1662-1677.

3. Darrah E, Rosen A, Giles JT, Andrade F: Peptidylarginine deiminase 2, 3 and 4 have distinct specificities against cellular substrates: novel insights into autoantigen selection in rheumatoid arthritis. Ann Rheum Dis 2012, 71(1):92-98.

4. Chang X, Hou X, Pan J, Fang K, Wang L, Han J: Investigating the pathogenic role of PADI4 in oesophageal cancer. Int J Bio/ Sci 2011, 7(6):769-781.

5. Wang $L$, Chang $X$, Yuan G, Zhao $Y$, Wang P: Expression of peptidylarginine deiminase type 4 in ovarian tumors. Int J Biol Sci 2010, 6(5):454-464.

6. Suzuki A, Yamada R, Chang $X$, Tokuhiro S, Sawada T, Suzuki M, Nagasaki M, Nakayama-Hamada M, Kawaida R, Ono M, Ohtsuki M, Furukawa H, Yoshino S, Yukioka M, Tohma S, Matsubara T, Wakitani S, Teshima R, Nishioka Y, Sekine A, lida A, Takahashi A, Tsunoda T, Nakamura Y, Yamamoto K: Functional haplotypes of PADI4, encoding citrullinating enzyme peptidylarginine deiminase 4, are associated with rheumatoid arthritis. Nat Genet 2003, 34(4):395-402.

7. Chang $X$, Han J: Expression of peptidylarginine deiminase type 4 (PAD4) in various tumors. Mol Carcinog 2006, 45(3):183-196.

8. Chang X, Fang K: PADI4 and tumourigenesis. Cancer Cell Int 2010, 10:7.

9. Zhang C, Fan L, Fan T, Wu D, Gao L, Ling Y, Zhu J, Li R, Wei L: Decreased PADI4 mRNA association with global hypomethylation in hepatocellular carcinoma during HBV exposure. Cell Biochem Biophys 2013, 65(2):187-195.

10. Lee TB, Park JH, Min YD, Kim KJ, Choi CH: Epigenetic mechanisms involved in differential MDR1 mRNA expression between gastric and colon cancer cell lines and rationales for clinical chemotherapy. BMC Gastroenterol 2008, 8:33.

11. Song J, Qu Z, Guo X, Zhao Q, Zhao X, Gao L, Sun K, Shen F, Wu M, Wei L: Hypoxia-induced autophagy contributes to the chemoresistance of hepatocellular carcinoma cells. Autophagy 2009, 5(8):1131-1144.

12. Petiot A, Ogier-Denis E, Blommaart EF, Meijer AJ, Codogno P: Distinct classes of phosphatidylinositol 3'-kinases are involved in signaling pathways that control macroautophagy in HT-29 cells. J Biol Chem 2000, 275(2):992-998.

13. Huesker M, Folmer $Y$, Schneider M, Fulda C, Blum HE, Hafkemeyer P: Reversal of drug resistance of hepatocellular carcinoma cells by adenoviral delivery of anti-MDR1 ribozymes. Hepatology 2002, 36(4 Pt 1):874-884.

14. Cuestas ML, Sosnik A, Mathet VL: Poloxamines display a multiple inhibitory activity of ATP-binding cassette $(A B C)$ transporters in cancer cell lines. Mol Pharm 2011, 8(4):1152-1164.

15. Toscano-Garibay JD, Aquino-Jarquin G: Regulation exerted by miRNAs in the promoter and UTR sequences: MDR1/P-gp expression as a particular case. DNA Cell Biol 2012, 31(8):1358-1364.

16. Leonard GD, Fojo T, Bates $S E$ : The role of $A B C$ transporters in clinical practice. Oncologist 2003, 8(5):411-424. 
17. Solazzo M, Fantappie O, Lasagna N, Sassoli C, Nosi D, Mazzanti R: P-gp localization in mitochondria and its functional characterization in multiple drug-resistant cell lines. Exp Cell Res 2006, 312(20):4070-4078.

18. Yoo BK, Chen D, Su ZZ, Gredler R, Yoo J, Shah K, Fisher PB, Sarkar D: Molecular mechanism of chemoresistance by astrocyte elevated gene-1. Cancer Res 2010, 70(8):3249-3258

19. Mizushima N, Noda T, Yoshimori T, Tanaka Y, Ishii T, George MD, Klionsky DJ, Ohsumi M, Ohsumi Y: A protein conjugation system essential for autophagy. Nature 1998, 395(6700):395-398.

20. Mizushima N, Sugita H, Yoshimori T, Ohsumi Y: A new protein conjugation system in human. The counterpart of the yeast Apg12p conjugation system essential for autophagy. J Biol Chem 1998, 273(51):33889-33892.

21. Klionsky DJ, Ohsumi Y: Vacuolar import of proteins and organelles from the cytoplasm. Annu Rev Cell Dev Biol 1999, 15:1-32.

22. Tang H, Da L, Mao Y, Li Y, Li D, Xu Z, Li F, Wang Y, Tiollais P, Li T, Zhao M: Hepatitis $B$ virus $X$ protein sensitizes cells to starvation-induced autophagy via up-regulation of beclin 1 expression. Hepatology 2009, 49(1):60-71.

23. Kroemer G, Jaattela M: Lysosomes and autophagy in cell death control. Nat Rev Cancer 2005, 5(11):886-897.

24. Levine B, Kroemer G: Autophagy in the pathogenesis of disease. Cell 2008, 132(1):27-42.

25. Levine B, Klionsky DJ: Development by self-digestion: molecular mechanisms and biological functions of autophagy. Dev Cell 2004, 6(4):463-477.

26. Klionsky DJ, Emr SD: Autophagy as a regulated pathway of cellular degradation. Science 2000, 290(5497):1717-1721.

27. Song YJ, Zhang SS, Guo XL, Sun K, Han ZP, Li R, Zhao QD, Deng WJ, Xie XQ Zhang JW, Wu MC, Wei LX: Autophagy contributes to the survival of CD133+ liver cancer stem cells in the hypoxic and nutrient-deprived tumor microenvironment. Cancer Lett 2013, 339(1):70-81.

28. Yu SW, Baek SH, Brennan RT, Bradley CJ, Park SK, Lee YS, Jun EJ, Lookingland KJ, Kim EK, Lee H, Goudreau JL, Kim SW: Autophagic death of adult hippocampal neural stem cells following insulin withdrawal. Stem Cells 2008, 26(10):2602-2610.

29. Gozuacik D, Kimchi A: Autophagy as a cell death and tumor suppressor mechanism. Oncogene 2004, 23(16):2891-2906.

30. Song J, Guo X, Xie X, Zhao X, Li D, Deng W, Song Y, Shen F, Wu M, Wei L: Autophagy in hypoxia protects cancer cells against apoptosis induced by nutrient deprivation through a Beclin1-dependent way in hepatocellular carcinoma. J Cell Biochem 2011, 112(11):3406-3420.

31. Wang Y, Li P, Wang S, Hu J, Chen XA, Wu J, Fisher M, Oshaben K, Zhao N, Gu Y, Wang D, Chen G, Wang Y: Anticancer peptidylarginine deiminase (PAD) inhibitors regulate the autophagy flux and the mammalian target of rapamycin complex 1 activity. J Biol Chem 2012, 287(31):25941-25953.

doi:10.1186/2045-3701-4-49

Cite this article as: Fan et al.: Peptidylarginine deiminase IV promotes the development of chemoresistance through inducing autophagy in hepatocellular carcinoma. Cell \& Bioscience 2014 4:49.

\section{Submit your next manuscript to BioMed Central and take full advantage of:}

- Convenient online submission

- Thorough peer review

- No space constraints or color figure charges

- Immediate publication on acceptance

- Inclusion in PubMed, CAS, Scopus and Google Scholar

- Research which is freely available for redistribution

Submit your manuscript at www.biomedcentral.com/submit
C Biomed Central 